Cahiers de recherches médiévales

Journal of medieval studies

12 spécial | 2005

Une ville, une destinée : Orléans et Jeanne d'Arc

\title{
Naudin Bouchard
}

Une carrière de fondeur orléanais pendant la guerre de Cent ans

Françoise Michaud-Fréjaville

\section{CpenEdition}

Journals

Édition électronique

URL : https://journals.openedition.org/crm/723

DOI : $10.4000 / \mathrm{crm} .723$

ISSN : 1955-2424

Éditeur

Honoré Champion

Édition imprimée

Date de publication : 1 juin 2005

Pagination : 75-84

ISSN : $1272-9752$

Référence électronique

Françoise Michaud-Fréjaville, " Naudin Bouchard », Cahiers de recherches médiévales [En ligne], 12 spécial | 2005, mis en ligne le 28 juin 2008, consulté le 15 décembre 2022. URL : http:// journals.openedition.org/crm/723; DOI : https://doi.org/10.4000/crm.723 


\title{
risM
}

\author{
$-7-$ \\ Naudin Bouchard, une carrière de fondeur orléanais \\ pendant la guerre de cent ans
}

L'importance que prit l'artillerie au cours de la guerre de Cent Ans permit à certains hommes de métier de manifester des capacités de reconversion et d'initiative dont on peut encore aujourd'hui retrouver la trace dans les documents et, dans certains cas, dans les résultats archéologiques ${ }^{1}$. Ainsi les fondeurs de cloches, détenteurs d'une technique très ancienne, très spécifique et, somme toute, très étroite, ont, dans de nombreux cas, su et peut-être, au fond, dû, adapter leur pratique des alliages, de la fusion et de la fonte non plus seulement à ces «saincts » de toute taille qui peuplaient clochers et beffrois, mais à des objets relativement nouveaux, au son beaucoup moins harmonieux : les canons.

Un des plus remarquables exemples de cette adaptation nous est fourni à Orléans par Naudin Bouchard, dont on peut, pour l'instant, suivre l'activité de façon à peu près continue entre 1396 et 1447 , soit un bon demi-siècle, ce qui est assez exceptionnel. Outre les nombreuses mentions qui parsèment les comptes de forteresse de la ville et évoquent les commandes livrées entre 1413 et 1430, et les comptes du pont dont il eut la charge, une trentaine d'actes environ le concernant sont lisibles dans les fonds notariaux comme dans les collections Joursanvault et Jarry ${ }^{2}$.

Naudin n'était peut-être pas originaire d'Orléans : la question n'est pas encore parfaitement claire. Sa famille est connue dans le Val de Loire à partir des dernières années du XIV ${ }^{\mathrm{e}}$ siècle, et lui-même ne nous est jamais signalé que comme "d'Orléans»; mais, en 1429, ses frères Etienne et Pierre, tous deux également «saintiers", étaient domiciliés à Tours ${ }^{3}$ et nous connaissons une activité tourangelle des Bouchard après que Naudin eut disparu de nos registres. Mais ce n'est pas si simple : Naudin et Pierre avaient vécu ensemble rue de la Clouterie (actuellement rue Etienne-Dolet), dans la paroisse Saint-Maurice-Saint-Éloi d'Orléans jusqu'en 1421 et, le 23 novembre de cette année-là, Naudin reconnaissait le travail de son frère «qui a demouré longuement avec icelui Naudin son frère et l'a servi de jour en jour en son dit mestier ${ }^{4}$, en lui concédant un certain nombre de biens immobiliers (c'est une manière de le payer sans puiser dans le capital en numéraire de

${ }^{1}$ Il ne reste pas de trace, pour l'instant, des travaux de N. Bouchard : les scories trouvées dans les fossés de la porte Bannier sont attribuées à la fin du XV siècle (voir Truelles et palissades, 10 ans d'archéologie à Orléans, Orléans, 1988, p. 91).

${ }^{2}$ Sauf précision particulière, les références sont celles des Archives départementales du Loiret. ${ }^{3} 3$ E 10135 (1 mi 253), 23 juin 1429, Pierre revend à Naudin son hôtel de la rue de la Closterie et Etienne prête 13 écus d'or à Naudin (voir infra). En 1433, Etienne Bouchard, saintier, est reçu à la confrérie de Saint-Gatien de Tours (B. Chevalier, Tours, ville royale, 1356-1520; origine et développement d'une capitale à la fin du Moyen Âge, Louvain-Paris, 1975, p. 139). ${ }^{4} 3$ E 10133, 23 novembre 1421 : le don entre vifs consistait en l' « habergement » de la Grange Mallet, une tuilerie à Saran et l'hôtel où demeurait Pierre, chargé de 6 1.t. de rente envers les héritiers de Jean Gouchet

Cahiers de Recherches Médiévales (XII -XV s.), 12spé, 2005 
l'entreprise), si bien qu'il n'est pas impossible que les Bouchard aient été véritablement originaires d'Orléans et se soient partagés entre les deux villes ligériennes au moment où les nécessités de la guerre élargissaient leur champ d'action.

\section{Le fondeur, des cloches aux canons}

Ce n'est cependant pas comme saintier que Naudin apparaît pour la première fois dans notre documentation: en 1396, le duc Louis lui fit refaire en cuivre les mesures officielles de la pinte $(1,12$ l.) et de la chopine (demi pinte) d'Orléans. La façon dont se présente la chose est d'ailleurs assez amusante : le duc, qui avait pris possession de son apanage en 1392 et souhaitait bien évidemment utiliser ses pouvoirs tout neufs, éprouva le besoin de faire préciser que la raison de cette commande n'était pas due à autre chose qu'à la vétusté extrême de ces objets : ils étaient «usés et en morceau»; néanmoins le mandant précisait bien que les nouvelles mesures seraient aux armes du duc ${ }^{5}$ !

La première cloche apparaît en février 1411 (n. st.); c'est la commande par le comte de Vendôme, Louis de Bourbon, d'une cloche de 3000 livres (environ une tonne et demi...) à livrer au port de Blois ${ }^{6}$. Ce qui est assez surprenant, c'est que la cloche n'ait pas été fondue au lieu où elle devait être pendue, ce qui était l'usage habituel. On peut imaginer le travail que représentait, même par voie d'eau, le transport d'une telle masse, et cette particularité nous empêche ici de savoir quelle était la destination finale de l'objet, sans doute un clocher de Vendôme. En revanche - et on le comprend ! - le fondeur se rendit à Chartres en 1414 pour y fondre «Gabriel», une des quatre cloches du clocher sud de la cathédrale et il en apporta la matière première d'Orléans ${ }^{7}$; cette cloche était nettement plus grosse (12 436 livres), mais proportionnellement moins chère que la précédente: le comte de Vendôme avait passé marché pour $360 \mathrm{l}$. t., les chanoines de la cathédrale pour 400 écus, soit 3001. t., différence que les talents en matière de marchandage des chanoines peuvent peutêtre expliquer.

Mais déjà la lutte entre Armagnacs et Bourguignons entraînait la reprise des armements urbains et la ville d'Orléans se préoccupait de la modernisation de sa défense : l'artillerie était désormais nécessaire. Faute de continuité dans la documentation, il est difficile de dresser un tableau absolument sûr de l'équipement dont s'était dotée la ville. Nous ne pouvons certifier que tous les canons et bombardes cités dans nos textes aient fonctionné en même temps. On arrive à un total de trentecinq ou trente-sept bouches à feu (avec des chambres supplémentaires) ${ }^{8}$ fournies par Naudin entre 1416 et 1429. Il fut en effet payé pour douze bombardes en 1417, pour

${ }^{5} 2$ J 21-134, N. Bouchard reçut 22 s. t. Rappelons les armes du duc Louis : de France au listel d'argent. Les armes de Philippe d'Orléans, le duc précédent, étaient de Valois. Entre temps, on avait pu reprendre celles du roi.

${ }^{6} 3$ E 10129. Pièce justificative I.

${ }^{7}$ C. Billot, Chartres à la fin du Moyen Âge, Paris, 1987, p. 23. Cette cloche fut refondue en 1498.

${ }^{8}$ F. Michaud-Fréjaville, «Une cité face aux crises: les remparts de la fidélité, de Louis d'Orléans à Charles VII, d'après les comptes de la forteresse d'Orléans ", dans Jeanne d'Arc, une époque, un rayonnement, Paris, 1981, p. 45, n. 2. 
dix-sept en 1418 (dont «une bombarde de cuivre appellee la Longue», de 373 livres) $)^{9}$, et trois en 1424. Quant aux canons, il en fondit trois en 1420 et un pendant le siège, «tres bel et tres long», pour mitrailler depuis le pont la bastille Saint-Privé ${ }^{10}$. Quelle part cela représentait-il du parc dont disposait la ville? Il est pratiquement impossible de le dire, mais c'en était vraisemblablement l'essentiel.

Comment apprit-il l'art de faire ces canons? Sans doute, comme ses collègues, par imitation des modèles achetés ailleurs, et en commençant par fabriquer des chambres supplémentaires pour des instruments qu'il n'avait pas fondus. Les premiers essais de canon datent en effet de l'été de 1410 avec du matériel acheté à Paris l'année précédente par le procureur Gilet Baudry ${ }^{11}$; la première bombarde «civique», si l'on peut dire, fut fondue en $1412^{12}$. Ensuite cela devint presque une routine. La ville d'Orléans, qui a connu finalement plutôt la paix entre 1356 et la guerre civile, était fort en retard, par exemple, sur les villes de Bretagne, obligées de se moderniser dès les années $1370^{13}$; mais, quand elle le fit, elle mit les bouchées doubles : elle avait été désarmée et "ouverte» en manière de répression après les émeutes fiscales de 1382 et il fallait rattraper le retard. Alors qu'à Rennes on ne fabriqua guère qu'une dizaine de pièces ente 1400 et $1440^{14}$, on en mit plus d'une trentaine en chantier sur nos bords de Loire. La ville capitale du duché n'étant pas d'ailleurs seule à devoir se défendre, nous retrouvons le fondeur équipant avant 1420 le château d'Yevre-le-Châtel d'au moins une pièce, un canon, avec ses deux boîtes de cuivre $^{15}$.

Avec les canons vont la poudre et le salpêtre. Naudin devint négociant: il fournit de la poudre dès $1413^{16}$, du salpêtre plus tard, lorsque les approvisionnements devinrent plus difficiles, en particulier quand les procureurs de la ville armagnacque n'eurent plus, après 1418, aucun rapport avec Paris aux mains des Bourguignons ${ }^{17}$.

${ }^{9}$ La bombarde, payée 8 1. t. le cent, coûta à la ville avec ses deux chambres, 29 1. 16s. 10 d. t. (CC 546, fol. 33).

${ }^{10} \mathrm{CC}$ 551, XLIIII : il pesait 443 livres et demi.

${ }^{11} \mathrm{CC} 542$, fol. 14 , pour les essais, fol. $20 \mathrm{v}$, pour la mention de l'achat. Le compte où figurait l'achat lui-même a disparu.

${ }^{12} \mathrm{CC} 543$, fol. 8 : Naudin a fait une chambre pour une bombarde nouvellement faite; le début du registre manquant, je ne peux affirmer, ce que je pense cependant, qu'il en avait fondu la partie principale.

${ }^{13}$ J.-P. Legay, "Travail du métal dans les villes armoricaines», dans Hommes et travail du métal, Paris, 1988, p. 191.

${ }^{14} \mathrm{~J} .-\mathrm{P}$. Legay, La ville de Rennes au $X V^{e}$ siècle à travers les comptes des miseurs, Paris, 1968, p. 280 et 294.

${ }^{15} 6 \mathrm{~J} 26,49$, la commande avait été passée avant 1418 : il s'agit dans ce document d'arrérages de paiement versés par le successeur d'Oudin Bernard (l'activité de ce dernier est connue jusqu'en 1418). La somme perçue par Naudin est de 65 1. t., elle vient en paiement du canon, des boîtes, de cordes d'arbalètes et d'un millier de traits.

${ }^{16} \mathrm{CC} 543$, XIII, fol. 8 : fourniture de 55 livres de poudre. En 1417, il vend 800 livres de poudre, CC, 545, XII, fol. 21.

${ }^{17}$ En 1416, c'est encore dans la capitale que se faisaient les achats : Jean Martin, procureur, y acheta 500 livres de salpêtre, 200 livres de soufre (CC 545, fol. 16v), et en 1417 les dernières arbalètes (CC 546, fol. 33) et du soufre vif (ibid., fol. 34v). 
Les quantités traitées par Naudin n'étaient pas médiocres : 1200 livres de salpêtre et 900 livres de poudre à canon en $1422^{18}$.

Cela ne signifiait pas pourtant l'abandon total des cloches. C'est encore à Naudin Bouchard que l'on s'adressa tout naturellement pour les quatre petits tocsins que l'on accrocha en 1420 aux portes de la ville. Dans des beffrois de bois furent accrochées une cloche de 107 livres, la plus grosse, au "pont» (en fait à la bastille Saint-Antoine), la suivante de 66 livres à la Porte Bernier, une autre de 55 livres à la porte Parisie, et la dernière, de 78 livres, à la porte Saint-Aignan ${ }^{19}$; ainsi quatre sons différents devaient permettre de savoir immédiatement de quel côté surgissait le danger, les habitants ayant entendu les essais effectués lors de la mise en place.

Nous ignorons le lieu où travaillait l'équipe de Bouchard. Traditionnellement les places et les fossés servaient d'atelier provisoire pendant le temps fort du moulage des canons, de même que le parvis ou l'entrée des églises pour les cloches. On sait que des scories de fonte qui semblent très nettement postérieures ont été retrouvées dans les fossés de la porte Bannier ${ }^{20}$. Pendant le siège, Naudin avait réussi à fondre un assez gros canon sans que nous sachions où il le coula ${ }^{21}$; mais ce ne saurait être dans les fossés qui subissaient alors le feu des bastilles anglaises ${ }^{22}$. La maison qu'il occupa en partie avec son frère, puis qu'il lui laissa et enfin qu'il lui racheta, était située dans le quartier des travailleurs du fer, entre deux artisans bien connus par les comptes de la ville: Guillaume Voisin et Guillaume Vaichot, tous deux «fêvres»; il n'est pas impossible qu'un atelier se soit trouvé là : l'artillerie médiévale n'est pas extrêmement volumineuse, les chambres ne nécessitent pas des moules de grande dimension. Dans ces instruments, ce qui tient le plus de place est la charpenterie sur laquelle ils sont montés, «maisons » des bombardes, " chevalets» des canons. À vrai dire le seul inconvénient des fonderies en ville était le risque d'incendie; or le quartier des métallurgistes était l'un des plus resserré de l'ancien castrum ; mais il faut croire que le talent et la vigilance des artisans était grande, car nul incendie accidentel important n'éclata jamais dans la cité.

Après les difficiles moments du siège, l'activité proprement manufacturière de Naudin se ralentit. Certes, on lui acheta encore la grande bassine à lessive où furent présentés au duc les 4000 écus d'or offerts par la ville lors de son entrée au retour de captivité ${ }^{23}$, mais l'industrieux saintier l'avait sans doute en magasin. Nau-

${ }^{18} \mathrm{CC} 548$, fol. 27.

${ }^{19} \mathrm{CC} 547$, fol. 51 , le tout fut payé 61 . t. pour 305 livres de poids, ce qui, par rapport à la cloche fournie au comte de Vendôme, montrerait une augmentation du prix de $60 \%$. En réalité nous sommes en pleine dévaluation monétaire (entre janvier 1419 et décembre 1420, la monnaie du dauphin Charles est passée du pied $24^{\mathrm{e}}$ au pied $216^{\mathrm{e}}$ ).

${ }^{20}$ Truelles et palissades, p. 91 . Les scories dateraient de la fin de la présence des fossés, soit des années 1480-90.

${ }^{21} \mathrm{Je}$ rectifie ici ce que j'ai laissé échapper dans «Fêvres et forgerons d'Orléans », Hommes et trevail du metal, p. 76, Naudin a bien fondu pendant le siège un canon «très bel et très long ", qu'on avait installé sur la partie du pont debout du côté de la ville : CC 551 (1435-1437), XLIII.

${ }^{22} \hat{A}$ moins, peut-être, d'utiliser le système particulier de la Tour Neuve qui disposait d'un fossé ou d'un glacis du côté de la ville.

${ }^{23} \mathrm{CC} 543$, XLIII, mandement du 22 mars 1441 (n. st.), versement de 24 s. parisis, pour ce «bacin a laver». 
din était devenu un notable et était sollicité par les tâches administratives que lui imposait la confiance du duc et des édiles qui en a fait un des trois responsables du pont et de l'hospice Saint-Antoine. Nous le retrouvons pourtant encore lorsque la cloche de la ville accrochée à Saint-Père-Empont se cassa en $1444^{24}$. Et peut-être prit-il auprès de lui un neveu pour l'aider, puisqu'il semble que le Guillaume Bouchart qui fondit, en 1456, la cloche de la Sainte-Chapelle de Châteaudun, venait d'Orléans $^{25}$. Néanmoins, le temps de l'artillerie est passée pour Bouchard, et il est le plus souvent mentionné dans les textes comme «bourgeois», ou encore "marchand», que comme saintier.

Une des dernières affaires où on peut le rencontrer se situe en 1445 : en août de cette année là, deux marchands venus de Dinant au pays de Liège, Gilles du Mont et Jean Bourrelier, "marchands de batterie» (nous dirions aujourd'hui «dinandiers »), lui ont laissé, devant témoins, trois fardeaux de poêles d'airain, des chaudrons et des chandeliers de cuivre qu'ils n'avaient pas réussi à vendre au cours de leur séjour et que Naudin se charge d'écouler dans la ville même. Deux mois plus tard, Naudin déclare avoir acheté le lot pour son compte ; malheureusement l'acte ne précise pas la somme versée, et encore moins l'usage que Naudin comptait faire de ces objets. La marchandise, qui n'avait pas trouvé preneur à Paris, vraisemblablement pour des raisons de concurrence, n'avait pas davantage intéressé les Orléanais, soit parce que la somme demandée était trop élevée, soit parce qu'elle était grevée de trop de taxes. On doit évidemment douter de la générosité du geste de Bouchard qui a bien dû y trouver son compte et peut-être même essayé de frauder en dissimulant «en manière de garde et de dépôt », ce qu'il avait d'ores et déjà dûment acquis ${ }^{26}$.

\section{Naudin Bouchard, bourgeois d'Orléans}

C'est donc au coeur de la vieille ville, dans la paroisse des travailleurs du métal, Saint-Éloi, que résidait la famille du fondeur. Nous savons qu'en 1412 il était marié avec une nommée Marguerite, et qu'il n'avait alors pas d'enfants : sa femme et lui se firent don mutuel de leurs biens, à charge de verser une rente de 20 l.t. annuels au père du conjoint décédé ; malheureusement nous ne savons pas où résidait le père de Naudin, Tours ou Orléans. On peut pencher pour la seconde solution, dans la mesure où la sœur de Naudin, Mahaut, déjà morte en 1421, était mariée à un Orléanais, Jean Aubour. Le fait que Naudin n'avait pas - ou pas encore - d'enfant est peut-être corroboré par la facilité avec laquelle il paya son frère Pierre par les donations qu'il lui fit de biens à Saran et en ville en $1421^{27}$. Sans doute aussi cela expli-

\footnotetext{
${ }^{24} \mathrm{CC} 554$.

${ }^{25}$ Renseignement communiqué par Claudine Billot.

${ }^{26} 3$ E 10149, mardi 7 septembre 1445, confirmation devant le Châtelet d'Orléans par Henri de la Coste, marchand d'Orléans, et Jean le Boulanger, marchand de baterie du pays de Liège, témoins du dépôt des objets en litige chez Naudin Bouchard. Ibid., 26 novembre 1445, Naudin reconnaît avoir acheté la marchandise « un certain prix et somme de deniers » quand Gillet du Mont était encore à Orléans.

${ }^{27}$ Pierre partit-il ensuite à Tours rejoindre leur autre frère Étienne? L'inscription d'une cloche de Saint-Aubin d'Angers semblerait le prouver: «Pierre Bouchard et Etienne son frere
} 
que-t-il ses largesses envers sa nièce - la fille de sa sœur-lorsque Naudin lui fit don cette même année, à l'occasion de son mariage, d'une maison, paroisse de l'AlleuSaint-Mesmin ${ }^{28}$. Un tableau de la fortune mobilière de Naudin est forcément lacunaire, faute d'avoir retrouvé son testament ou, surtout, un partage (pour l'instant on ignore si sa femme lui a survécu). En l'état actuel, on sait qu'il avait des ressources d'origine variée :

- des maisons : au moins deux à Orléans même, paroisses de Saint Maurice (Saint-Éloi) et de L'Alleu-Saint-Mesmin (jusqu'en 1421); une «masure» paroisse Saint Laurent ${ }^{29}$; un hôtel à la Chapelle Saint Mesmin ${ }^{30}$; plus tard il a fait l'acquisition de «l'ostel de l'Image Saint-Martin» à Cléry, qu'il louait pour partie en 1447 à Etienne Masle ${ }^{31}$;

- un ensemble à Saran, composé d'une maison, d'une grange et d'une tuilerie, donné à bail ${ }^{32}$; une autre «masure » et places rénovées après le siège ${ }^{33}$; des prés dans cette même paroisse ${ }^{34}$;

- une propriété rurale, de dimensions modestes : un «habergement» à Damberon en Beauce, « dite la grange Mallet $»^{35}$;

\footnotetext{
/ M'ont de leurs mains faict de ceste maniere / Quand la Pucelle Anglois conquit / l'an mil quatre cent vingt et neuf », cité par B. Chevalier, Tours, ville royale, p. 138.

${ }^{28} 3$ E 10133, 13 novembre 1421: «icelui Naudin confirma que, au traité de Jehan Le Paige, boucher marchand d'Orliens, et de Jehanne, fille de Jehan Oubour et de feu Mahaut,jadis sa fame, seur Naudin [...], il donna et quicte et delaisse [...] a ladicte Jehanne, sa niepce [...] une maison sise en la rue du [ill.] en la paroisse de Laleu Saint-Mesmin d'Orliens, tenant a l'ostel de Guillaume Chenu d'une part, et a l'ostel de Harvet darriere, pour tenir a XL s. de rente a tousjoursmais, due chascun an a Pierre de Saint-Mesmin. Item, ung ostel, grenier et appentis [...] avec trois arpens de vigne et trois arpens de terre, a XXVIII s. de rente, que ledit Naudin avoit assis sur les heritaiges de feu Thenot Belin, tous situés a Sandillon ». En cas de décès de Jeanne sans enfants, le tout reviendra à Naudin et à ses propres héritiers.

${ }^{29} 3 \mathrm{E} 10131,11$ juin 1412 , achat d'une masure paroisse saint-Laurent.

${ }^{30} 3 \mathrm{E} 10135,29$ mai 1430, Il le donne à bail pour un an à Denise la Fromagelle, veuve de Jehan Fromageau, à charge de la réparer (y aurait-il eu des dégâts à la suite du siège ?).

${ }^{31} 3$ E 10141, 30 janvier 1446 (1447). La location comprenait également les étables et la moitié du jardin.

${ }^{32} 3$ E 10133,29 octobre 1421. Pièce justificative $n^{\circ} 2$.

${ }^{33} 3$ E 10135, dimanche 12 février 1429 (1430). Guillaume Pynault et Jeanne sa femme vendent à Naudin, saintier d'Orléans, une masure et place couverte à neuf, sur le chemin de Paris, paroisse de Saran, chargés de 6 d. t de cens envers le chapitre de Sainte-Croix.

${ }^{34} 2 \mathrm{~J} 2387,10$ juillet 1426 , achat de 5 quartiers de pré au clos près du Deffoys, à Saran ; $3 \mathrm{E}$ 10133, 15 novembre 1421, achat pour 30 1. t. à Alice, veuve de Jean Rahez, à présent femme de Jean Bourguignon, d'un arpent de pré clos d'épines, au clos de la Poterie, chargé de 6 d. t. de cens envers le chapitre cathédral.

${ }^{35} 3$ E 10135, 12 décembre 1429, bail de 6 ans à Simon Fraicon de Damberon, d'un habergement, vignes, terres et appartenances, moyennant 2 muids de blé « tel come il croistra par tiers a la mesure de la moison", le premier tiers étant toutefois en avoine. La vigne devra être travaillée "doulcement et raisonnablement de toutes façons et provignee jusqu'aux raisins coupés »; le bailleur paiera les travaux $32 \mathrm{~s}$. p. par an et il fournira les charnes (pieux) et osiers que le preneur viendra chercher à Orléans «ou plus pres si faire se peut » (Damberon, com., can. Orgères, arr. Châteaudun, Eure-et-Loir). Cet « habergement » est cité dans la donation à Pierre en 1421, il est alors dit des acquêts de Naudin.
} 
- un clos de vigne, paroisse Saint-Privé ${ }^{36}$.

L'immobilier n'est pas son seul investissement et il semble bien que, de temps en temps, il ait pris des dîmes à ferme ${ }^{37}$ et qu'il ait acheté des rentes ${ }^{38}$; vraisemblablement il s'agissait de prêts plus ou moins avoués.

Finalement, ce patrimoine demeure modeste. Excellent praticien, Naudin n'est pas un capitaine d'industrie, ni un ambitieux. Il jouit d'une excellente réputation et c'est pourquoi il fut un des trois bourgeois auxquels on confia la remise en état du pont après le siège ${ }^{39}$, charge qu'il garda plusieurs années et dont une étude a précisé la façon dont ses collègues et lui assumèrent les responsabilités ${ }^{40}$. Mais c'était, semble-t-il, un homme assez sage, peut-être parce qu'il n'avait pas d'héritier direct ${ }^{41}$. Peut-être aussi était-ce un trait de famille. Bernard Chevalier trace en effet d'Etienne et Jean Bouchard, frères ou neveux de notre saintier, un portrait très proche de notre Orléanais : ils pratiquent la fonte, ils sont fondeurs habiles, faiseurs de chandeliers de cuivre; Etienne, canonnier du roi, est aussi l'auteur du "gros sainct» de Saint-Gatien ; Jean est, en outre, «marchand publicque et va par les villes et marchiez pour faire son mestier... et est bien hérité en la ville de Tours $»^{42}$. Il y eut, certes, une dynastie Bouchard, mais elle resta, peut-on dire, très provinciale.

Quand, parvenu à un âge respectable - actif dès 1396, il devait avoir au moins soixante-dix ans en 1446-, Naudin donne en location son hôtel semi-rural de Cléry ; il se réserve une moitié de jardin et le droit d'aller et venir à sa guise dans ses vignes derrière la maison; dans cette demeure, où il ne doit pouvoir plus guère aller, il laisse les meubles, ceux d'une modeste maison des champs : cinq lits, trois tables, deux coffres dont un fermant à clef, une paire de chenets de fer, de quoi passer un ou deux jours en apportant les repas ou en allant dans une des auberges de la petite ville. "L'ostel de l'Image Saint-Martin» était-il, pour Naudin, une halte près d'un pèlerinage, un lieu de réflexion pour un vieillard à l'ombre de Notre-Dame ${ }^{43}$ ?

${ }^{36} 3 \mathrm{E}$ 10144, 26 décembre 1436 (bail à façon).

${ }^{37} 3$ E 10131,6 juillet 1413.

${ }^{38}$ Dès 1409 , Naudin reconnaît toucher 18 muids de froment et 18 s. p. de rente $(3$ E 10129, 18 avril 1409); bien plus tard, on trouve la mention d'une autre rente d'un muids de méteil, due par Thomas Bouiloie et Perrette la Bigle, sa femme, de Darvoy, et dont les arrérages s'élevaient à 10 1. t. (3 E 10149, 10 novembre 1445).

${ }^{39} 3$ E 10135, nomination du 28 juin 1430, en compagnie d'Henri Moreau et Jean Guyonneau.

${ }^{40} \mathrm{~A}$. Gasnier, L'cuvre du pont d'Orléans et de l'hopital Saint-Antoine d'après les registres de comptes (1386-1437), Mémoire de maîtrise, dactyl., Tours, 1990.

${ }^{41}$ Le Guillaume Bouchard, saintier d'Orléans, qui fit une cloche pour Châteaudun en 1456 était-il un fils ou un neveu de Naudin? Il n'a pas joué à Orléans le rôle de son parent. Étienne Bouchard fut appelé depuis Tours en 1453 à la rescousse «pour avoir son opinion", lorsque Robin Boivin "faillit a fondre» la cloche de l'Hôtel des Crénaux, nouvel hôtel de ville (CC 556, fol. 43v). Dans cette affaire, Guillaume Bouchard est étrangement absent et dans la difficile mise au place de clochettes du carillon, Guillaume n'apparaît cité qu'une fois (CC 556, fol. 46).

${ }^{42} \mathrm{~B}$. Chevalier, Tours ville royale, p. 138, n. 105.

${ }^{43} 3$ E 10149, 30 janvier 1446 (1447), bail à Étienne Masle, avec une mention marginale signalant le reçu au terme de Noël des 161 . p. de location (deuxième terme prévu à la Saint-Jean) et une seconde signalant la mise à néant du bail, le 22 juin 1447, or le bail était triennal. Si j'osais, je suggérerais que Naudin n'a pas supporté l'idée de ne plus être le maître dans cette 
Le vent de modernité que, dans les années 1440-1450, Jacques Cœur faisait souffler, entre autres sur le marché des armes, n'ébranle pas, semble-t-il, nos fondeurs. Ils restent à leur poste, solides comme leur production, mais dans une tradition de fort sobre et industrieuse bourgeoisie.

maison à l'époque du pélerinage ; seul le testament de Bouchard (non retrouvé) pourrait nous dire s'il avait effectivement une dévotion particulière envers Notre-Dame de Cléry. 


\title{
Pièces justificatives
}

\begin{abstract}
$\mathrm{N}^{\circ} 1$ : commande à Naudin Bouchard d'une cloche par Louis de Bourbon comte de Vendôme (A. D. Loiret, 3 E 10129, lundi 24 février 1410 (1411).

Naudin Bouchard, saintier, marchand d'Orleans, contracte et marchande a noble et puissant seigneur, Monseigneur Loys de Bourbon, conte de Vendosme, de lui faire mouler bien et seurement une cloche du poyds de trois milliers ou environ, et icelle cloche rendue preste, duement ouvree de son mestier de saintier a ses cousts et perils, au port de Bloys, dedens la Saint Germain prouchaine venant, pour le pris de six vins livres tournois chascun millier, et se ladite cloche poise deux ou trois cens plus desdits trois milliers, ledit Naudin sera payé au dit pris de $\mathrm{VI}^{\mathrm{xx}} 1 . t$. le millier. Et sur ce ledit Naudin a confessé avoir eu et receu de mondit seigneur le conte la somme de trois cens livres tournois, dont il s'est tenu a content et en a quitté ledit monseigneur le Conte et touz autres. Promettant ledit Naudin non venir contre ledit contrat et icelle cloche faire et rendre deuement comme dit est [...].
\end{abstract}

$\mathrm{N}^{\circ} 2$ : bail d'une tuilerie à Saran (A. D. Loiret, 3 E 10133, 29 octobre 1421).

Robin de Longemeau, maçon, demourant en la paroisse de Saran prant de Naudin Bouchart, saintier, marchant d'Orlians, qui lui baille de rente ferme ou pension de la Toussains prochaine venant jusque a LIX ans prochains ensuivants les heritaiges qui ensuivent, que ledit Naudin a naguere achetés dudit Robin, ou il demoure, assis ou clos de Villepau en ladite paroisse de Saran. C'est assavoir ung hostel a demourer, une tieullerie, l'ourouer et habillement de tieullerie, avec un arpent de vigne et cinq arpens et demi de terre appartenant au dit hostel tenuz a cens du chappitre Saint Aignan d'Orlians, a tenir pour quatre milliers de tieulle garniz d'enfestaux, tout bon et convenable, pris sur ladite tieullerie, de rente ferme ou pension, a paier chacun an aux termes de Penthecoste et de la mi-aoust, a chacun terme la moitié, dont le premier terme sera a la Penthecoste prouchaine venant. Et par ce faisant ledit preneur, ses hoirs et autres seront tenuz desdits hostel, tieullerie, fours, ouvrouer et habillements soustenir, et desdits heritaiges faire, et de ouvrer en ladite tieullerie diligement et deuement durant lesdits LIX ans et en la fin lesser en bon estat et convenable; et de cependant paier du leur, oultre ladite rente, ledit cens ou nom dudit bailleur et de ses hoirs et en acquitant et gardant. Et outre ce, seront tenus lesdits preneur, ses hoirs et [ayant droits] de ladite tieullerie faire couvrir de tieulle dedans ung an prouchainement venant, en lui baillant par ledit bailleur, IIII seules de III toises, lates et chanlates qu'il y fauldra pour ladite couverture, et de, audit lieu, faire ung puis bon et convenable dedens ung an, en lui baillant et paient par ledit bailleur XX 1.t. et IIII journees a voiturer de ses chevaulx ou beufs. Et ou cas que ledit preneur, ses hoirs et [ayant droits] deffauldront de payer la dite rente ou de ouvrer de la dite tieullerie par ung an ensuivant, ledit bailleur, ses hoirs et [ayant droits] pourront prandre et espletter et faire leur prouffit desdits lieux sans contredit 
et eulx faire paier les arrerages de la dite rente et des interests. Et s'ils vendent ou transportent ledit droit que ont oudit bien et esdits heritaiges, celui ou ceulx qui ledit droit vouldront tenir seront tenuz eulx et obligés oudit tenement audit bailleur, comme est ledit preneur dedens ung an aprés ledit transport ou mutacion ou non ledit bailleur. 